\title{
Tissue engineering and its potential use in surgery
}

\author{
L.-P. Kamolz, H. Koch, C. Kasper
}

Published online: 5 June 2013

(C) Springer-Verlag Wien 2013

The loss or failure of an organ or tissue is one of the most frequent, devastating, and costly problems in healthcare. Current treatment modalities include transplantation of organs, surgical reconstruction, use of mechanical devices, or supplementation of metabolic products. A new field, tissue engineering, applies the principles and methods of engineering, material science, and cell and molecular biology toward the development of viable substitutes, which restore, maintain, or improve the function of human tissues. In this special issue, we outline the opportunities and challenges of this emerging interdisciplinary field and its attempts to provide solutions to tissue creation and repair in different fields of surgery.

To date, surgical strategies that have evolved to deal with tissue loss include organ transplantation from one individual to another, tissue transfer from a healthy site to the affected site in the same individual, and replacement of tissue function with mechanical devices such as prosthetic valves/joints and dialysis machines. Additional medical strategies include pharmacologic supplementation of the metabolic products of absent or nonfunctional tissue. While these strategies incorporate significant advances in the field of medicine, they have a number of inherent limitations as follows: supplementing of metabolic products of lost or diseased organs is currently most commonly used in endocrinology. Hormonal products, such as insulin can be chronically supplemented. The

L.-P. Kamolz M.D., M.Sc. $(\bowtie) \cdot$ H. Koch M.D.

Division of Plastic, Aesthetic and Reconstructive Surgery,

Research Unit for Tissue Regeneration, Repair and Reconstruction, Department of Surgery, Medical University of Graz,

Auenbruggerplatz 29, $8036 \mathrm{Graz}$, Austria

e-mail: lars.kamolz@medunigraz.at

C. Kasper Ph.D.

Institute of Applied Microbiology, University of Natural Resources and Life Sciences Vienna, Vienna, Austria lack of normal feedback mechanisms, though, may result in imbalance of hormone levels, causing acute (hypo- or hyperglycemic crisis) or long-term complications. Nonbiological materials are used routinely in ophthalmology (intraocular lenses), orthopedic surgery (replacement of joints), cardiovascular surgery (mechanical heart valves, vascular prostheses, stents), and reconstructive and cosmetic surgery. These materials (stainless steel, Dacron, polyacrylates, etc.) are also used for extracorporeal techniques, such as dialysis or plasmapheresis. Major complications include infection, lack of biocompatibility (acute thrombosis, chronic irritation, carcinogenicity) and limited material durability. The important limitation is using implantable devices in pediatric patients_repeated surgery may be required as the patient undergoes his or her pubertal growth spurt. Surgical reconstruction such as using different organs (colon to replace esophagus or saphenous vein as a coronary bypass graft) or unaffected tissue (flaps in many variations or free grafts) to replace diseased tissue or organ, also has its limitations. The replacement organs consist of a different tissue type that cannot properly function in the new environment and are unable to completely replace lost function. Morbidity at the donor site is a major concern. Organ transplantation of kidney, liver, heart, lung, and pancreas have made many innovative advances in recent years. Unfortunately, however, it is severely limited by an increasing donor shortage. The other major problems are high cost of the procedures and the necessity of lifelong immunosuppression with its serious side effects. Tissue engineering, on the other hand, seeks to provide a new solution to tissue loss. The term tissue engineering was introduced officially at a workshop of the National Science Foundation 1988 [1] and was defined as:

“...the application of principles and methods of engineering and life sciences toward fundamental understanding of structure-function relationship in normal and pathological mammalian tissues and the devel- 
opment of biological substitutes to restore, remain or improve tissue function". Thus, tissue engineering aims at the optimization of tissue or organ function with constructs that contain specific population of living cells. Unlike traditional approaches for the treatment of lost tissue or organ function, tissue engineering holds the promise to one day replace living tissue with living tissue that is designed and constructed to meet the needs of each individual patient [2-5]. In effect, cells could be isolated from a healthy site in the patient, expanded in vitro and/or modified by gene therapy to replace a defective gene, and then reimplanted back into the patient in the appropriate location under appropriate conditions to correct the functional defect. And here, the surgeon can potentially adapt these fundamental techniques of tissue engineering with the tools of minimally invasive surgery to bring the whole concept of disease treatment to a new higher and modern technological level. Three avenues have been explored in creating new tissues: the injection of isolated cells, the development of encapsulated systems (closed systems), and the transplantation of cells in matrices (open systems). Tissue engineering is more than an emerging newcomer, and encouraging results are already reported. The need is enormous and the potential benefits immeasurable. However, much work needs still to be done. One of the more important biological questions that must be addressed is how can a given cell population be accessed and then expanded? The signals governing cell growth in culture are becoming better understood, but vary somewhat with the specific cell type, that is, skeletal muscle satellite cells proliferate rapidly while cardiac myocytes do not divide at all in culture; moreover, hepatocytes in culture proliferate poorly, though the liver shows a remarkable capacity for regeneration in vivo. In addition, a great deal of work has been performed over the past few years to isolate stem cell populations for a variety of tissues. Although the use of stem cell cultures may provide an almost limitless sup- ply of cells for transplantation, it will first be necessary to isolate the stem cell and confirm that daughter cells can indeed be directed to differentiate appropriately. Furthermore, closer investigations towards homogeneity of isolated stem cell populations are of utmost importance. The isolation of specific subpopulations and investigation of their specific "features" and characteristics of these may contribute to better understanding of cell growth during expansion and lead to optimized culture processes including protocols mimicking more physiological conditions during cultivation. Regardless of the cell source, how can the differentiated function of cells be promoted once cells are transplanted? These and other fundamental issues demand close cooperation among clinical scientists, biologists, materials engineers, and chemists to meet the inherent challenges of tissue engineering [2-5]. The aim of this special issue is to give an overview of current possibilities concerning tissue engineering in different surgical fields.

\section{Conflict of interest}

The author declares that there is no conflict of interest.

\section{References}

1. Skalak R, Fox CF. Tissue engineering. Granlibakken, Lake Tahoe: Proc workshop. New York: Liss; 1988. p. 26-29.

2. Chapekar MS. Tissue engineering: challenges and opportunities. J Biomed Mater Res. 2000;53(6):617-20.

3. Terada S, Sato M, Sevy A, Vacanti JP. Tissue engineering in the twenty-first century. Yonsei Med J. 2000;41(6):685-91.

4. Griffith LG, Naughton G. Tissue engineering-current challenges and expanding opportunities. Science. 2002;295(5557):1009-14.

5. Fisher MB, Mauck RL. Tissue engineering and regenerative medicine: recent innovations and the transition to translation. Tissue Eng Part B Rev. 2013;19(1):1-13. 